\title{
EXPLICIT SUMMATION OF THE CONSTITUENT WKB SERIES AND NEW APPROXIMATE WAVE FUNCTIONS
}

\section{VLADIMIR V. KUDRYASHOV AND YULIAN V. VANNE}

Received 6 December 2001 and in revised form 11 April 2002

The independent solutions of the one-dimensional Schrödinger equation are approximated by means of the explicit summation of the leading constituent WKB series. The continuous matching of the particular solutions gives the uniformly valid analytical approximation to the wave functions. A detailed numerical verification of the proposed approximation is performed for some exactly solvable problems arising from different kinds of potentials.

\section{Introduction}

Perturbation theory, the variational method and the WKB approximation are very extensively used in quantum mechanics. If we deal with perturbation theory or with the variational method then similar questions arise. How to find the unperturbed Hamiltonian or how to find the trial function for an arbitrarily given potential? Universal answers are absent. In this sense both mentioned methods are incomplete. In contrast, the WKB approximation is directly determined by a given potential. However the conventional WKB approximation has unphysical singularities. An old problem in semiclassical analysis is the development of global uniform approximations to the wave functions. In previous works $[6,7]$, an essential improvement of the WKB approach was introduced for the logarithmic derivatives of the wave functions. In the present paper, we construct the second-order continuous approximation to the wave functions. The quality of the approximate wave functions is verified by means of a comparison with the exact solutions for different kinds of potentials. 
We consider the linear one-dimensional Schrödinger equation

$$
\frac{d^{2} \Psi(q, \hbar)}{d q^{2}}=\frac{Q(q)}{\hbar^{2}} \Psi(q, \hbar)
$$

where $Q(q)=2 m(V(q)-E)$ for an arbitrary potential $V(q)$. The logarithmic derivative

$$
Y(q, \hbar)=\frac{d \ln \Psi(q, \hbar)}{d q}
$$

of a wave function $\Psi(q, \hbar)$ satisfies the nonlinear Riccati equation

$$
\frac{d Y(q, \hbar)}{d q}+(Y(q, \hbar))^{2}=\frac{Q(q)}{\hbar^{2}} .
$$

The WKB approach deals just with functions $Y(q, \hbar)$. In this approach, two independent solutions $Y^{ \pm}(q, \hbar)$ of the Riccati equation are represented by their asymptotic expansions

$$
Y_{\text {as }}^{ \pm}(q, \hbar)=\hbar^{-1}\left( \pm Q^{1 / 2}+\sum_{n=1}^{\infty} \hbar^{n} Y_{n}^{ \pm}(q)\right)
$$

in powers of Plank's constant $\hbar$. The usual WKB approximation contains a finite number of leading terms $Y_{n}^{ \pm}(q)$ from the complete expansions $Y_{\text {as }}^{ \pm}(q, \hbar)$. This approximation is not valid at turning points where $Q(q)=0$.

As it is well known, the WKB series is divergent. Numerous references regarding asymptotic expansions may be found in [4]. The direct summation of a divergent series does not exist. By summing one means finding a function to which this series is the asymptotic expansion [3]. In recent years many studies have been devoted to extracting some useful information about the exact eigenfunctions from the divergent WKB series (see, e.g., [5] and the references therein). There are several investigations on the properties of the WKB terms $[2,11]$. Unlike entirely exact but very complicated methods for some classes of potentials (see, e.g., [12]) our new way of using the WKB series gives an approximate but very simple and universal method of solving the Schrödinger equation.

\section{Explicit summation of the constituent WKB series}

Since [7] is likely to be inaccessible for the large majority of readers we reproduce previous results. First of all, the analysis of the well-known 
recursion relations $[3,2]$

$$
Y_{n+1}^{ \pm}=-\left(2 Y_{0}^{ \pm}\right)^{-1}\left(\sum_{j=1}^{n} Y_{j}^{ \pm} Y_{n+1-j}^{ \pm}+d Y_{n}^{ \pm} / d q\right), \quad Y_{0}^{ \pm}= \pm Q^{1 / 2}
$$

shows that the WKB terms are of the form

$$
Y_{n}^{ \pm}(q)=Q^{(1-3 n) / 2} \sum_{j=1}^{n} A_{n, j}^{ \pm}\left(Q^{\prime}(q), Q^{\prime \prime}(q), \ldots, Q^{(j)}(q)\right) Q^{j-1},
$$

where $Q^{\prime}(q)=d Q(q) / d q, Q^{\prime \prime}(q)=d^{2} Q(q) / d q^{2}$, and $Q^{(j)}(q)=d^{j} Q(q) / d q^{j}$. Second, the substitution of (2.2) into (1.4) allows us to reconstruct the asymptotic WKB series as an infinite sum

$$
Y_{\mathrm{as}}^{ \pm}(q, \hbar)= \pm \hbar^{-1} Q^{1 / 2}+\sum_{j=1}^{\infty} Z_{\mathrm{as}, j}^{ \pm}(q, \hbar)
$$

of new constituent (partial) asymptotic series

$$
Z_{\mathrm{as}, j}^{ \pm}(q, \hbar)=\left(\hbar^{2 / 3}\right)^{j-2} \sum_{n=j}^{\infty}\left(\frac{Q}{\hbar^{2 / 3}}\right)^{j-(3 n+1) / 2} A_{n, j}^{ \pm}\left(Q^{\prime}(q), Q^{\prime \prime}(q), \ldots, Q^{(j)}(q)\right)
$$

in powers of the ratio $Q / \hbar^{2 / 3}$. With the help of the recursion relations (2.1) we derive simple expressions

$$
A_{n, 1}^{ \pm}=\left(Q^{\prime}\right)^{n} B_{n, 1}^{ \pm}, \quad A_{n, 2}^{ \pm}=Q^{\prime \prime}\left(Q^{\prime}\right)^{n-2} B_{n, 2}^{ \pm}
$$

for two leading sequences of coefficients $A_{n, j}^{ \pm}$. Here the numbers $B_{n, 1}^{ \pm}$are determined by the following recursion relations:

$$
B_{n+1,1}^{ \pm}=\mp\left(\frac{1}{2} \sum_{k=1}^{n} B_{k, 1}^{ \pm} B_{n+1-k, 1}^{ \pm}+\frac{1-3 n}{4} B_{n, 1}^{ \pm}\right), \quad n \geq 1, B_{1,1}^{ \pm}=-\frac{1}{4},
$$

and $B_{n, 2}^{ \pm}$is connected with $B_{n, 1}^{ \pm}$as follows:

$$
B_{n, 2}^{ \pm}=-\frac{2}{5} n B_{n, 1}^{ \pm}, \quad n \geq 2 .
$$

The complete series $Y_{\mathrm{as}}^{ \pm}(q, \hbar)$ are approximated by a finite number of leading constituent series $Z_{\mathrm{as}, j}^{ \pm}(q, \hbar)$ in contrast to the use of a finite number of leading terms $Y_{n}^{ \pm}(q)$ in the conventional WKB approach. If 
we can find functions $Z_{j}^{ \pm}(q, \hbar)$ which are represented by asymptotic expansions $Z_{\mathrm{as}, j}^{ \pm}(q, \hbar)$, then we obtain new approximations to the solutions of the Riccati equation. The number of used constituent series corresponds to the order of a proposed approximation. For instance the expressions $\pm \hbar^{-1} Q^{1 / 2}+Z_{1}^{ \pm}(q, \hbar)$ are interpreted as the first-order approximations. In this paper, we consider only the second-order approximations $\pm \hbar^{-1} Q^{1 / 2}+Z_{1}^{ \pm}(q, \hbar)+Z_{2}^{ \pm}(q, \hbar)$.

Introducing the dimensionless variable

$$
a(q, \hbar)=\frac{1}{\hbar^{2 / 3}} \frac{Q(q)}{\left|Q^{\prime}(q)\right|^{2 / 3}}
$$

we are able to rewrite the leading constituent expansions in the form

$$
\pm \hbar^{-1} Q^{1 / 2}+Z_{\mathrm{as}, 1}^{ \pm}(q, \hbar)+Z_{\mathrm{as}, 2}^{ \pm}(q, \hbar)=\frac{1}{\hbar^{2 / 3}} \frac{Q^{\prime}}{\left|Q^{\prime}\right|^{2 / 3}} y_{\mathrm{as}, 1}^{ \pm}(a)+\frac{Q^{\prime \prime}}{Q^{\prime}} y_{\mathrm{as}, 2}^{ \pm}(a),
$$

where we separate the asymptotic series in $a$

$$
\begin{aligned}
& y_{\mathrm{as}, 1}^{ \pm}(a)= \pm a^{1 / 2}+\sum_{n=1}^{\infty} B_{n, 1}^{ \pm} a^{1-(3 n+1) / 2}, \\
& y_{\mathrm{as}, 2}^{ \pm}(a)=\sum_{n=2}^{\infty} B_{n, 2}^{ \pm} a^{2-(3 n+1) / 2} .
\end{aligned}
$$

The leading terms

$$
\begin{gathered}
\pm a^{1 / 2}-\frac{1}{4} a^{-1}, \\
\pm \frac{1}{8} a^{-3 / 2}+\frac{9}{32} a^{-3}
\end{gathered}
$$

of these series may be deduced by using (2.6) and (2.7).

Our aim is to sum constituent series (2.10). In other words, we must find functions $y_{j}^{ \pm}(a)$ which are represented by these expansions. In order to perform the identification we substitute the approximate function

$$
Y_{\text {ap }}^{ \pm}(q, \hbar)=\frac{1}{\hbar^{2 / 3}} \frac{Q^{\prime}}{\left|Q^{\prime}\right|^{2 / 3}} y_{1}^{ \pm}(a)+\frac{Q^{\prime \prime}}{Q^{\prime}} y_{2}^{ \pm}(a)
$$


into the Riccati equation (1.3). As a result we get the following equations:

$$
\begin{gathered}
\frac{d y_{1}^{ \pm}}{d a}+\left(y_{1}^{ \pm}\right)^{2}=a, \\
\frac{d y_{2}^{ \pm}}{d a}+2 y_{1}^{ \pm} y_{2}^{ \pm}=\frac{1}{3}\left(2 a \frac{d y_{1}^{ \pm}}{d a}-y_{1}^{ \pm}\right),
\end{gathered}
$$

for the functions $y_{j}^{ \pm}(a)$. Direct verification shows that the asymptotic expansions (2.10) satisfy these equations.

Equation (2.14) is the Riccati equation for the logarithmic derivatives of linear combinations of the well-studied Airy functions $\operatorname{Ai}(a)$ and $\operatorname{Bi}(a)$ [1]. We select particular solutions by means of the known asymptotics (2.11). In the classically allowed region where $Q(q)<0(a<0)$ we derive the explicit expressions

$$
y_{1}^{ \pm}(a)=\frac{d}{d a} \ln (\operatorname{Bi}(a) \mp i \operatorname{Ai}(a))
$$

and in the classically forbidden region where $Q(q)>0(a>0)$ we get the other solutions

$$
\tilde{y}_{1}^{-}(a)=\frac{d}{d a} \ln \operatorname{Ai}(a), \quad \tilde{y}_{1}^{+}(a)=\frac{d}{d a} \ln \operatorname{Bi}(a) .
$$

Finally, we can obtain the solutions of the linear equation (2.15) with asymptotics (2.12) in the closed form

$$
\begin{aligned}
& y_{2}^{ \pm}(a)=\frac{1}{30}\left[-8 a^{2}\left(y_{1}^{ \pm}(a)\right)^{2}-4 a y_{1}^{ \pm}(a)+8 a^{3}-3\right], \\
& \tilde{y}_{2}^{ \pm}(a)=\frac{1}{30}\left[-8 a^{2}\left(\tilde{y}_{1}^{ \pm}(a)\right)^{2}-4 a \tilde{y}_{1}^{ \pm}(a)+8 a^{3}-3\right] .
\end{aligned}
$$

Although the functions (2.16), (2.17), and (2.18) have the asymptotic expansions (2.10) if $|a|$ is large it should be stressed that the obtained functions possess different expansions if $|a|$ is small. Replacing $y_{j}^{ \pm}$by $\tilde{y}_{j}^{ \pm}$ in expression (2.13) we get the second pair

$$
\tilde{Y}_{\mathrm{ap}}^{ \pm}(q, \hbar)=\frac{1}{\hbar^{2 / 3}} \frac{Q^{\prime}}{\left|Q^{\prime}\right|^{2 / 3}} \tilde{y}_{1}^{ \pm}(a)+\frac{Q^{\prime \prime}}{Q^{\prime}} \tilde{y}_{2}^{ \pm}(a)
$$

of approximate solutions.

It is not surprising that the asymptotics of our approximation coincide with the WKB asymptotics far away from the turning points. At the 
same time our approximation reproduces the known [3] satisfactory approximation near the turning points. Naturally, our approximation gives the exact result for the linear potential $V(q)=k q$ of a uniform field. Note that this potential represents an example of the explicit summation of the WKB series for the logarithmic derivative of a wave function.

\section{Approximate wave functions for the two-turning-point problem}

With the aid of the uniformly valid approximation to solutions of the Riccati equation derived in the preceding section, we can now construct approximate wave functions. We consider the problem with two real turning points $q_{-}$and $q_{+}\left(q_{+}>q_{-}\right)$. The potential has its minimum at point $q_{m}$. The first and second derivatives of the smooth potential are continuous at point $q_{m}$.

Two pairs of independent solutions of the Schrödinger equation are approximated by functions

$$
\begin{aligned}
& \Psi_{\mathrm{ap}}^{ \pm}(q)=\exp \left(\int Y_{\mathrm{ap}}^{q}\left(q^{\prime}\right) d q^{\prime}\right), \\
& \tilde{\Psi}_{\mathrm{ap}}^{ \pm}(q)=\exp \left(\int^{q} \tilde{Y}_{\mathrm{ap}}^{ \pm}\left(q^{\prime}\right) d q^{\prime}\right) .
\end{aligned}
$$

In accordance with the requirements of quantum mechanics, we must retain only the decreasing solutions $\tilde{\Psi}_{\text {ap }}^{-}(q)$ in the classically forbidden regions $\left(q<q_{-}\right.$and $\left.q>q_{+}\right)$. In the classically allowed region $\left(q_{-}<q<\right.$ $\left.q_{+}\right)$, we retain a linear combination of two oscillatory solutions $\Psi_{\text {ap }}^{+}(q)$ and $\Psi_{\text {ap }}^{-}(q)$. By matching particular solutions at the turning points $q$ and $q_{+}$, we obtain the continuous approximate wave function which is represented by the following formulas:

$$
\Psi_{1}(q)=C \cos \frac{\pi}{3} \exp \left(-\int_{q}^{q_{-}} \tilde{Y}_{\mathrm{ap}}^{-}\left(q^{\prime}\right) d q^{\prime}\right)
$$

if $q<q_{-}$,

$$
\begin{aligned}
\Psi_{2}(q)= & C \exp \left(\int_{q_{-}}^{q} \frac{Y_{\mathrm{ap}}^{+}\left(q^{\prime}\right)+Y_{\mathrm{ap}}^{-}\left(q^{\prime}\right)}{2} d q^{\prime}\right) \\
& \times \cos \left(\int_{q_{-}}^{q} \frac{Q^{\prime}}{\left|Q^{\prime}\right|} \frac{Y_{\mathrm{ap}}^{+}\left(q^{\prime}\right)-Y_{\mathrm{ap}}^{-}\left(q^{\prime}\right)}{2 i} d q^{\prime}-\frac{\pi}{3}\right)
\end{aligned}
$$


if $q_{-}<q<q_{+}$, and

$$
\begin{aligned}
\Psi_{3}(q)= & C(-1)^{n} \cos \frac{\pi}{3} \exp \left(\int_{q_{+}}^{q} \tilde{Y}_{\mathrm{ap}}^{-}\left(q^{\prime}\right) d q^{\prime}\right) \\
& \times \exp \left(\int_{q_{-}}^{q_{+}} \frac{Y_{\mathrm{ap}}^{+}\left(q^{\prime}\right)+Y_{\mathrm{ap}}^{-}\left(q^{\prime}\right)}{2} d q^{\prime}\right)
\end{aligned}
$$

if $q>q_{+}$.

Here we have the new quantization condition

$$
\int_{q_{-}}^{q_{+}} \frac{Q^{\prime}}{\left|Q^{\prime}\right|} \frac{Y_{\mathrm{ap}}^{+}(q, E)-Y_{\mathrm{ap}}^{-}(q, E)}{2 i} d q=\pi\left(n+\frac{2}{3}\right), \quad n=0,1,2, \ldots
$$

which determines the spectral value $E_{\mathrm{sp}}(n)$ of energy implicitly. We denote the wave functions with $E=E_{\mathrm{sp}}(n)$ as $\Psi_{\mathrm{ap}}(q, n)$. Then we may choose the value of an arbitrary constant $C$ in order to ensure the usual normalization $\left\langle\Psi_{\text {ap }}(n) \mid \Psi_{\text {ap }}(n)\right\rangle=1$ where $\left|\Psi_{\text {ap }}(n)\right\rangle$ is the vector in Hilbert space which corresponds to the function $\Psi_{\text {ap }}(q, n)$. The proposed approximation is an alternative to the well-known $[3,10]$ Langer approximation [9] which employs an $\hbar$-expansion different from the WKB series.

Thus the approximate eigenfunctions are determined completely. However a question arises regarding the optimal approximate eigenvalues, because the value $E_{\mathrm{sp}}(n)$ is not a unique choice.

Since explicit expressions for wave functions have already been obtained, we are able to calculate the expectation values

$$
\bar{E}(n)=\left\langle\Psi_{\text {ap }}(n)|\hat{H}| \Psi_{\text {ap }}(n)\right\rangle
$$

of the Hamiltonian

$$
\hat{H}=-\frac{\hbar^{2}}{2 m} \frac{d^{2}}{d q^{2}}+V(q) .
$$

In accordance with the eigenvalue problem

$$
\hat{H}|\Psi\rangle-E|\Psi\rangle=0
$$

we construct the discrepancy vector

$$
|D(e, n)\rangle=\hat{H}\left|\Psi_{\text {ap }}(n)\right\rangle-e\left|\Psi_{\text {ap }}(n)\right\rangle,
$$

where $e$ is an arbitrary parameter while $\hat{H}$ and $\left|\Psi_{\text {ap }}(n)\right\rangle$ are given. It is natural to require that the discrepancy vector should not contain a 
component proportional to the approximate eigenvector. In other words, we consider the orthogonality condition

$$
\left\langle\Psi_{\text {ap }}(n) \mid D(e, n)\right\rangle=\bar{E}(n)-e=0
$$

as a criterion for the selection of the optimal approximate eigenvalue. As a result we just get $\bar{E}(n)$ while $E_{\mathrm{sp}}(n)$ does not fulfil the above requirement. It should also be noted that the scalar product $\langle D(e, n) \mid D(e, n)\rangle$ is minimized at $e=\bar{E}(n)$.

\section{Verification of the proposed approximation}

Now we must verify our approximation numerically for exactly solvable problems. We compare the normalized approximate wave functions $\Psi_{\text {ap }}(q, n)$ with the normalized exact wave functions $\Psi_{\mathrm{ex}}(q, n)$.

In order to estimate the closeness of two functions $f_{1}(q)$ and $f_{2}(q)$, we consider two corresponding vectors $\left|f_{1}\right\rangle$ and $\left|f_{2}\right\rangle$ in Hilbert space. Then we construct a deviation vector $|\Delta f\rangle=\left|f_{1}\right\rangle-\left|f_{2}\right\rangle$ and a scalar product

$$
\langle\Delta f \mid \Delta f\rangle=\left\langle f_{1} \mid f_{1}\right\rangle+\left\langle f_{2} \mid f_{2}\right\rangle-\left\langle f_{1} \mid f_{2}\right\rangle-\left\langle f_{2} \mid f_{1}\right\rangle
$$

Now we can define the relative deviation

$$
\delta f=1-\frac{\left\langle f_{1} \mid f_{2}\right\rangle+\left\langle f_{2} \mid f_{1}\right\rangle}{\left\langle f_{1} \mid f_{1}\right\rangle+\left\langle f_{2} \mid f_{2}\right\rangle}
$$

as a numerical estimate of the closeness of two functions. Note that $\delta f=$ 0 if $f_{1}(q)=f_{2}(q)$.

Thus, we get the following estimate:

$$
\delta \Psi(n)=1-\left\langle\Psi_{\mathrm{ex}}(n) \mid \Psi_{\mathrm{ap}}(n)\right\rangle
$$

in the case of the normalized real functions $\Psi_{\text {ex }}(q, n)$ and $\Psi_{\text {ap }}(q, n)$. The same numerical comparison may be performed

$$
\delta \Psi^{\prime}(n)=1-\frac{2\left\langle\Psi_{\mathrm{ex}}^{\prime}(n) \mid \Psi_{\mathrm{ap}}^{\prime}(n)\right\rangle}{\left\langle\Psi_{\mathrm{ex}}^{\prime}(n) \mid \Psi_{\mathrm{ex}}^{\prime}(n)\right\rangle+\left\langle\Psi_{\mathrm{ap}}^{\prime}(n) \mid \Psi_{\mathrm{ap}}^{\prime}(n)\right\rangle}
$$

for the first derivatives $\Psi^{\prime}(q)=d \Psi(q) / d q$. Naturally, we can define analogous estimates for higher derivatives. 
Moreover, we estimate the closeness of functions $\hat{H} \Psi_{\text {ap }}(q, n)$ and $\hat{H} \Psi_{\text {ex }}(q, n)$ with the help of the following quantity:

$$
\delta \hat{H} \Psi(n)=\frac{\left\langle\Psi_{\mathrm{ap}}(n)\left|\hat{H}^{2}\right| \Psi_{\mathrm{ap}}(n)\right\rangle+E_{\mathrm{ex}}(n)^{2}\left(1-2\left\langle\Psi_{\mathrm{ex}}(n) \mid \Psi_{\mathrm{ap}}(n)\right\rangle\right)}{\left\langle\Psi_{\mathrm{ap}}(n)\left|\hat{H}^{2}\right| \Psi_{\mathrm{ap}}(n)\right\rangle+E_{\mathrm{ex}}(n)^{2}},
$$

where $E_{\text {ex }}(n)$ is the exact energy value.

In addition, we compare two functions $\hat{H} \Psi_{\text {ap }}(q, n)$ and $\bar{E}(n) \Psi_{\text {ap }}(q, n)$. This comparison may be performed when we do not know the exact solutions. As a result we get the relative discrepancy

$$
d(n)=\frac{\left\langle\Psi_{\mathrm{ap}}(n)\left|\hat{H}^{2}\right| \Psi_{\mathrm{ap}}(n)\right\rangle-\bar{E}(n)^{2}}{\left\langle\Psi_{\mathrm{ap}}(n)\left|\hat{H}^{2}\right| \Psi_{\mathrm{ap}}(n)\right\rangle+\bar{E}(n)^{2}}
$$

which is directly connected with the Schrödinger equation under consideration.

Finally, we characterize our approximation by the usual relative energy error

$$
\triangle E(n)=\frac{\bar{E}(n)}{E_{\mathrm{ex}}(n)}-1 .
$$

The verification is performed for three potentials with different asymptotics. They are the harmonic oscillator potential

$$
V(q)=k q^{2}
$$

the Morse potential

$$
V(q)=\frac{r^{2} \hbar^{2} \alpha^{2}}{2 m}\left(e^{-2 \alpha q}-2 e^{-\alpha q}\right),
$$

and the modified Pöschl-Teller potential

$$
V(q)=\frac{\lambda(\lambda-1) \hbar^{2} \alpha^{2}}{2 m \cosh ^{2}(\alpha q)}
$$

The exact solutions of the Schrödinger equation for these potentials may be found in [8].

Table 4.1 shows that our approximation gives fairly accurate results for all considered potentials and for all considered quantities. Hence, the performed reconstruction of the WKB series and subsequent explicit summation of the leading constituent (partial) series yield the satisfactory (qualitative and quantitative) description of wave functions. 
274 Explicit summation of the constituent WKB series

TABLE 4.1. Numerical verification of the proposed approximation.

\begin{tabular}{llllll}
\hline$n$ & $\delta \Psi(n)$ & $\delta \Psi^{\prime}(n)$ & $\delta \hat{H} \Psi(n)$ & $d(n)$ & $\Delta E(n)$ \\
\hline \multicolumn{5}{c}{ The harmonic oscillator potential } \\
\hline 0 & $2.16 \cdot 10^{-4}$ & $4.60 \cdot 10^{-3}$ & $5.79 \cdot 10^{-2}$ & $5.32 \cdot 10^{-2}$ & $4.52 \cdot 10^{-3}$ \\
1 & $1.59 \cdot 10^{-5}$ & $7.12 \cdot 10^{-5}$ & $1.33 \cdot 10^{-4}$ & $6.09 \cdot 10^{-5}$ & $5.64 \cdot 10^{-5}$ \\
2 & $3.86 \cdot 10^{-6}$ & $1.05 \cdot 10^{-5}$ & $1.48 \cdot 10^{-5}$ & $3.78 \cdot 10^{-6}$ & $7.15 \cdot 10^{-6}$ \\
3 & $1.50 \cdot 10^{-6}$ & $3.10 \cdot 10^{-6}$ & $4.12 \cdot 10^{-6}$ & $7.31 \cdot 10^{-7}$ & $1.89 \cdot 10^{-6}$ \\
\hline \multicolumn{5}{c}{ The Morse potential $(\gamma=4.5)$} \\
\hline 0 & $2.76 \cdot 10^{-4}$ & $5.98 \cdot 10^{-3}$ & $4.59 \cdot 10^{-3}$ & $5.77 \cdot 10^{-3}$ & $-1.45 \cdot 10^{-3}$ \\
1 & $4.95 \cdot 10^{-5}$ & $2.34 \cdot 10^{-4}$ & $2.45 \cdot 10^{-4}$ & $3.56 \cdot 10^{-4}$ & $-1.60 \cdot 10^{-4}$ \\
2 & $2.26 \cdot 10^{-5}$ & $8.22 \cdot 10^{-5}$ & $3.72 \cdot 10^{-4}$ & $4.51 \cdot 10^{-4}$ & $-1.01 \cdot 10^{-4}$ \\
3 & $1.77 \cdot 10^{-5}$ & $6.43 \cdot 10^{-5}$ & $2.35 \cdot 10^{-3}$ & $2.49 \cdot 10^{-3}$ & $-1.59 \cdot 10^{-4}$ \\
\hline \multicolumn{5}{c}{ The modified Pöschl-Teller potential $(\gamma=5)$} & \\
\hline 0 & $2.31 \cdot 10^{-4}$ & $6.60 \cdot 10^{-3}$ & $4.49 \cdot 10^{-3}$ & $5.70 \cdot 10^{-3}$ & $-1.44 \cdot 10^{-3}$ \\
1 & $2.91 \cdot 10^{-5}$ & $2.10 \cdot 10^{-4}$ & $1.65 \cdot 10^{-4}$ & $2.97 \cdot 10^{-4}$ & $-1.62 \cdot 10^{-4}$ \\
2 & $1.98 \cdot 10^{-5}$ & $6.17 \cdot 10^{-5}$ & $2.36 \cdot 10^{-4}$ & $3.18 \cdot 10^{-4}$ & $-1.01 \cdot 10^{-4}$ \\
3 & $5.22 \cdot 10^{-5}$ & $4.90 \cdot 10^{-5}$ & $1.95 \cdot 10^{-3}$ & $2.05 \cdot 10^{-3}$ & $-1.48 \cdot 10^{-4}$ \\
\hline
\end{tabular}

\section{References}

[1] M. Abramovitz and I. A. Stegun (eds.), Handbook of Mathematical Functions, Dover Publications, New York, 1970.

[2] C. M. Bender, K. Olaussen, and P. S. Wang, Numerological analysis of the WKB approximation in large order, Phys. Rev. D 16 (1977), no. 6, 1740-1748.

[3] C. M. Bender and S. A. Orszag, Advanced Mathematical Methods for Scientists and Engineers, International Series in Pure and Applied Mathematics, McGraw-Hill, New York, 1978.

[4] J. P. Boyd, The devil's invention: asymptotic, superasymptotic and hyperasymptotic series, Acta Appl. Math. 56 (1999), no. 1, 1-98.

[5] E. Delabaere, H. Dillinger, and F. Pham, Exact semiclassical expansions for one-dimensional quantum oscillators, J. Math. Phys. 38 (1997), no. 12, 61266184.

[6] V. V. Kudryashov, Quantum systems, New Trends and Methods (Y. S. Kim et al., eds.), World Scientific, Singapore, 1997, pp. 202-205.

[7] - Reconstruction of the WKB series as a new method for the approximate solution of the Schrödinger equation, Dokl. Nats. Akad. Nauk Belarusi 42 (1998), no. 6, 45-49 (Russian).

[8] L. D. Landau and E. M. Lifshitz, Quantum Mechanics, Pergamon Press, Oxford, 1977.

[9] R. E. Langer, On the connection formulas and the solutions of the wave equation, Phys. Rev. 51 (1937), no. 8, 669-676.

[10] F. W. J. Olver, Asymptotics and Special Functions, Computer Science and Applied Mathematics, Academic Press, New York, 1974. 
[11] M. Robnik and V. G. Romanovski, Some properties of WKB series, J. Phys. A 33 (2000), no. 28, 5093-5104.

[12] A. Voros, Exact resolution method for general 1D polynomial Schrödinger equation, J. Phys. A 32 (1999), no. 32, 5993-6007.

Vladimir V. Kudryashov: Institute of Physics, National Academy of Sciences of Belarus, 68 F. Skaryna Avenue, 220072 Minsk, Belarus

E-mail address: kudryash@dragon.bas-net.by

Yulian V. Vanne: Department of Chemistry, University of Konstanz, Fach M721

D-78457, Konstanz, Germany

E-mail address: yulian.2.vanne@uni-konstanz.de 


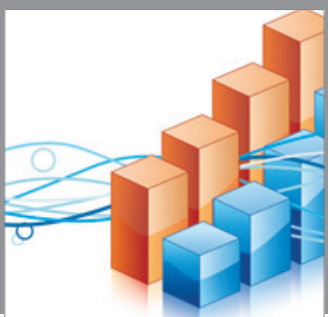

Advances in

Operations Research

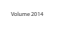

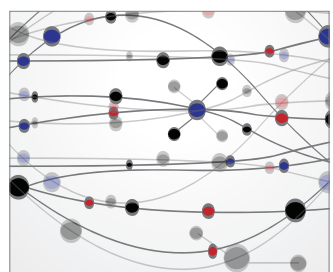

\section{The Scientific} World Journal
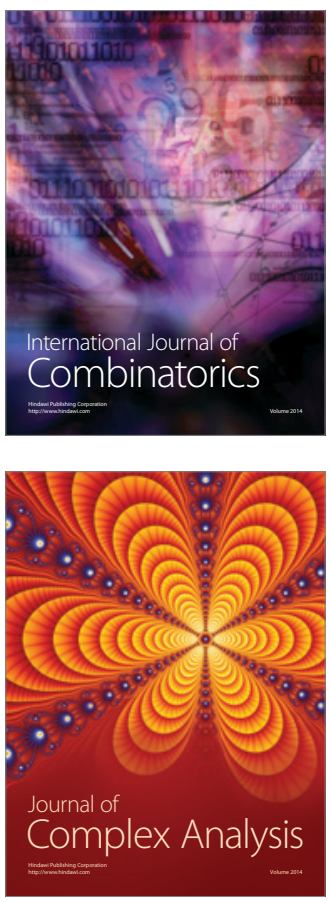

International Journal of

Mathematics and

Mathematical

Sciences
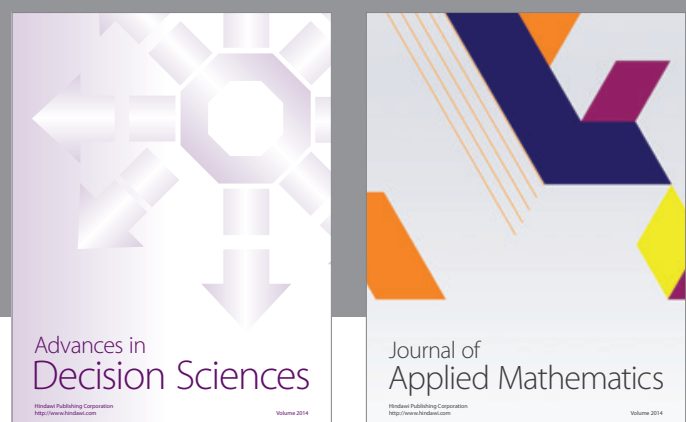

Journal of

Applied Mathematics
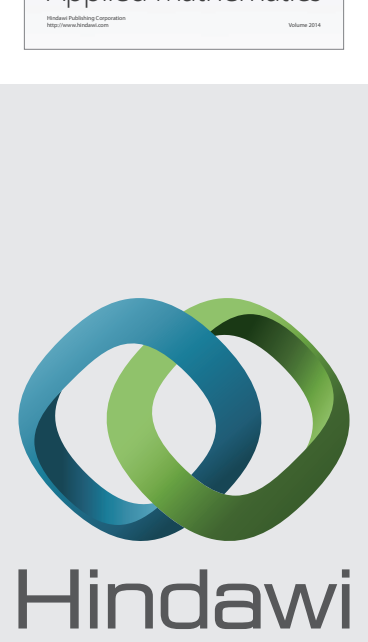

Submit your manuscripts at http://www.hindawi.com
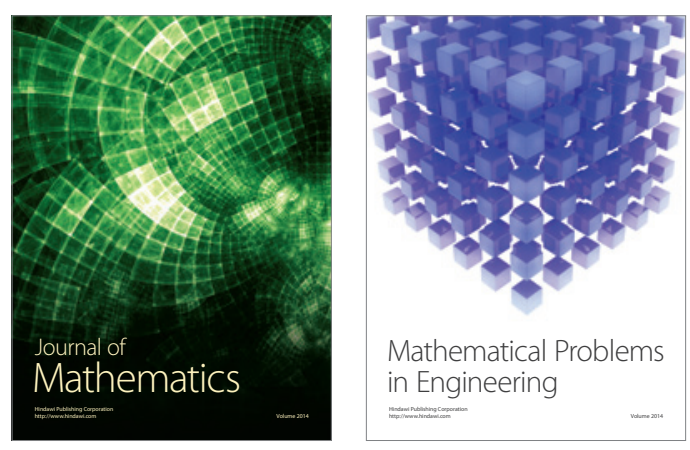

Mathematical Problems in Engineering
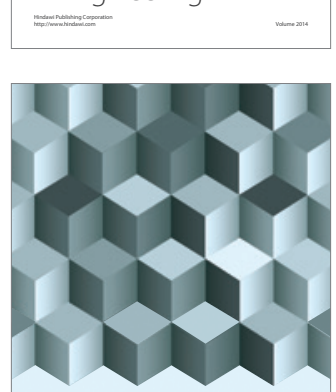

Journal of

Function Spaces
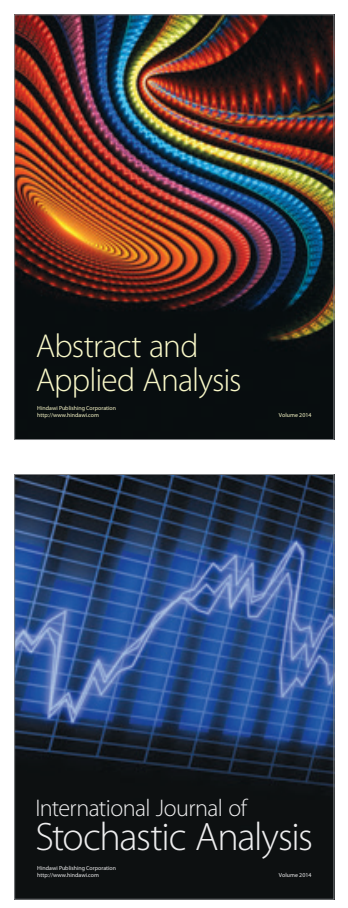

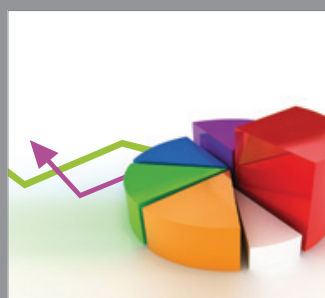

ournal of

Probability and Statistics

Promensencen
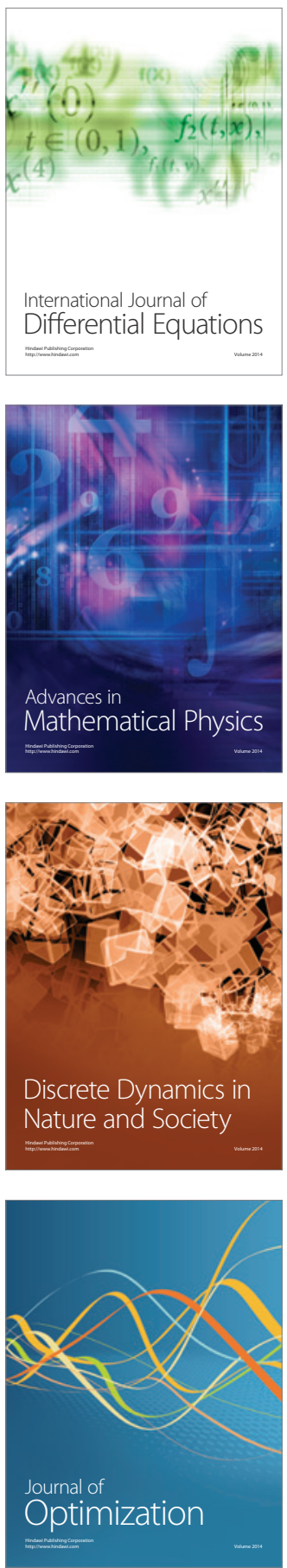